\section{FDA approves antitoxin antibody}

The FDA has approved Merck \& Co's bezlotoxumab to reduce the recurrence of Clostridium difficile infection, marking the first approval for a new approach to the treatment of bacterial infections. Unlike antibiotics, which kill pathogenic bacteria, bezlotoxumab is an antibody that mops up toxins that are released by $C$. difficile.

Although antibiotics can control C. difficile, the infection recurs within weeks in around $25 \%$ of patients. In two phase III trials that tested bezlotoxumab in conjunction with standard of care antibiotics, Merck showed that their adjunct antibody reduced recurrence to $16-17 \%$ of patients. The most common adverse reactions included nausea, pyrexia and headache.

Analysts expect the market for bezlotoxumab to be modest, at US $\$ 212$ million per year in worldwide sales by 2020. However, the approval could boost interest in the growing pipeline of antibodies for the treatment of bacterial infections (Nat. Rev. Drug Discov. 14, 737-738; 2015).

In some cases these antibodies, like bezlotoxumab, act on bacterial toxins. AstraZeneca's phase Il candidate MEDI4893 targets the Staphylococcus aureus alpha toxin, for example, and Bellus Health's phase II shigamab targets the Escherichia coli shiga toxin type 2. Other antibodies target the bacteria more directly. AstraZeneca's phase II candidate MEDI3902, for example, targets the exopolysaccharide Psl and the secretion protein PcrV on the body of Pseudomonas aeruginosa bacteria.

One benefit of these targeted antibodies is that they might be less likely to induce broad resistance among bacteria or disturb the healthy microbiome.

Asher Mullard

\section{PCSK9 pipeline}

Promising phase II data from The Medicines Company's inclisiran have paved the way for phase III trials of a long-acting oligonucleotide-based PCSK9 inhibitor. Although the FDA has already approved two PCSK9 blockers - Amgen's evolocumab and Sanofi and Regeneron's alirocumab - these subcutaneously delivered lipid-lowering antibodies are administered every 2-4 weeks for the treatment of hyperlipidaemia. If data hold up, inclisiran might only require two or three injections per year.

The Medicines Company presented the new data at the American Heart Association (AHA) Scientific Sessions 2016 in New Orleans in November. Preliminary data from the first 189 patients in a phase II study of the RNA interference (RNAi)-based drug showed that one injection reduced low-density lipoprotein cholesterol (LDL-C) levels by $59 \%$ compared with placebo at day 60 , and that two injections reduced LDL-C by $57 \%$ at day 120 . Treatment was well tolerated. There was one death and one case of increased liver enzyme levels, but investigators said neither were related to treatment.

But even as The Medicines Company prepared to move its proprotein convertase subtilisin/kexin type 9 (PCSK9) programme forward, Pfizer discontinued development of its would-have-been competitor bococizumab. The antibody had been in phase III trials in more than 27,000 patients. Safety data showed, however, that it was more immunogenic than the competition. Pfizer also cited "the evolving treatment and market landscape", referencing disappointing sales for the approved PCSK9 inhibitors.

Payers are pushing back against the high cost of these drugs - around US $\$ 14,000$ per patient per year - because of the absence as yet of definitive data proving that they reduce cardiovascular events.

The field is awaiting the results of large cardiovascular outcomes studies that could open up the market. Another study that was presented at that AHA meeting provided some encouragement. Researchers dosed 968 patients with evolocumab or statins, and used ultrasound to measure the size of atherosclerotic plaques, a risk factor for heart attacks. Plaques shrank in $64 \%$ of evolocumab patients, and in $47 \%$ of statin-treated patients.

Asher Mullard

\section{EMA opens its data vaults}

European regulators have started publishing the full clinical trial reports for drugs that are approved for use in the
European Union. Results can be accessed at https://clinicaldata.ema.europa.eu/web/ cdp/home.

As Nature Reviews Drug Discovery went to press, the agency had released data for only two drugs: Onyx Pharmaceuticals' carfilzomib and AstraZeneca's lesinurad. The combined reports for these two drugs alone are 260,000 pages long. The European Medicines Agency (EMA) plans to publish clinical data on 2-4 products per month. Once it has cleared a backlog of publishable reports, it will release data for new drugs within 60 days of approval decisions. It will also release data for drugs that are withdrawn before a decision is made.

"Patients and clinicians have been waiting a long time for clinical trial data," said Yann Le Cam, Chief Executive Officer of EURORDIS - Rare Diseases Europe and member of the EMA's management board. "Access to this new knowledge base can help to accelerate innovation by reducing duplication of research and de-risking some new developments."

Asher Mullard

\section{Access to medicines report cards}

Drug companies are getting moderately better at enabling access to drugs for people in lowand middle-income countries shows a recent report from the non-profit Access to Medicine Foundation.

GlaxoSmithKline topped the biennial ranking, for the 5 th consecutive time, followed by Johnson \& Johnson and Novartis.

Companies are also doing a better job of addressing global health priorities, says the report. The top 20 pharmaceutical companies are developing 420 products for the most burdensome diseases of low- and middle-income countries, up by more than 100 products since the 2014 Index. "We see evidence that collaborative RED models are engaging the industry in developing urgently needed medicines they would not otherwise be considering because there is not enough of a commercial market for them," said Jayasree lyer, Executive Director of the Access to Medicine Foundation.

The report also found that good practice is limited to a narrow range of products and countries, providing plenty of room for improvement.

Asher Mullard 